\title{
PROGRESS IN THE CONCEPTUAL DESIGN OF THE ISAC-II LINAC AT TRIUMF
}

\begin{abstract}
R. E. Laxdal, R. Lee, M. Pasini, TRIUMF, Abstract

TRIUMF is funded through to 2005 to proceed with an extension to the ISAC facility, ISAC-II, to permit acceleration of radioactive ion beams up to energies of at least 6.5 MeV/u for masses up to 150 . The upgrade includes the addition of a post-stripper superconducting linac to accelerate beams of $3 \leq A / q \leq 7$ to an energy of at least 6.5 MeV/u. The possibility of using the superconducting linac to accelerate multiple charge states has been investigated. We have in particular studied both the transverse and the longitudinal phase space for three different lattices, doublet, triplet and solenoid, focusing our attention on developing a lattice to increase the multi-charge acceptance. The present linac cavity parameters, concept scenarios and beam dynamic simulation results will be presented.
\end{abstract}

\section{INTRODUCTION}

Federal funding for the ISAC-II project [1] is now in place as part of the TRIUMF five year funding cycle from April 2000 - April 2005. The project will see the final energy of the post-accelerator increased to at least $6.5 \mathrm{MeV} / \mathrm{u}$ and the mass-range extended up to 150 .

A schematic of the ISAC-I/ISAC-II layout is shown in Fig. 1. A room-temperature $\mathrm{IH}$ structure operating in $\mathrm{cW}$ mode is used to accelerate the ions of $A \leq 30$ from $0.15-0.4 \mathrm{MeV} / \mathrm{u}$. The beam is then stripped and charge selected. Ions with $3 \leq A / q \leq 7$ are matched into a superconducting DTL, accelerated to at least $6.5 \mathrm{MeV} / \mathrm{u}$ and then transported to the experimental stations. The short independently phased cavities that make up the post-stripper linac give a wide velocity acceptance and so the final energy for each ion can be optimized.

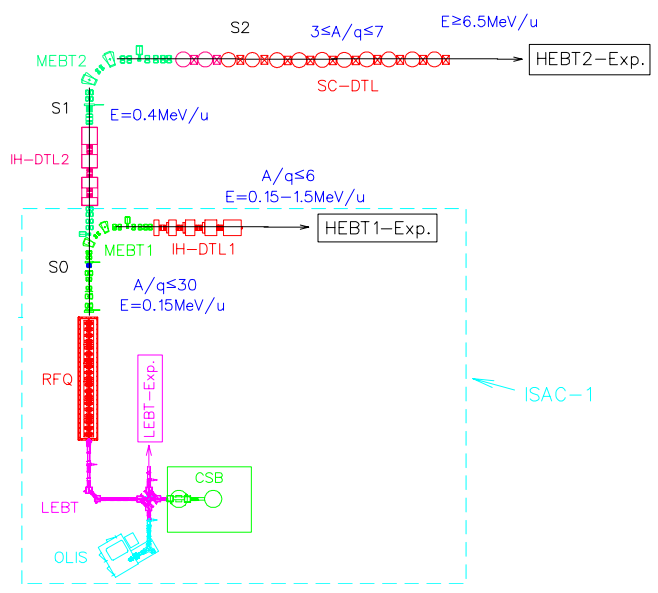

Figure 1: Schematic of the ISAC-I/ISAC-II accelerator layout.
Recently the American RIA project has utilized the idea of reducing accelerator length hence cost by adding multiple stripping stations and maintaining beam intensity by accelerating multiple charge states [3] . We consider the possibility of utilizing multi-charge acceleration in the ISAC-II superconducting linac[4].

\section{CAVITY MODELLING}

The specification of the superconducting linac calls for three cavity types of frequency $\left(\beta_{o}\right) 70.7 \mathrm{MHz}(4.2 \%)$, $106.08 \mathrm{MHz}(7.2 \%)$ and $141.4 \mathrm{MHz}(10.5 \%)$ respectively delivering an effective accelerating voltage of $42.7 \mathrm{MV}$. The medium $\beta$ quarter wave bulk Niobium cavity[2] is shown in Fig. 2 along with some design parameters.

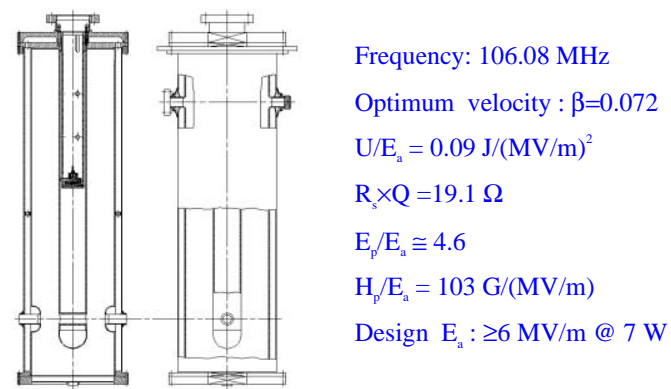

Figure 2: The prototype $106.1 \mathrm{MHz}$ medium- $\beta$ cavity for the ISAC-II project at TRIUMF.

To simplify cryostat design all cavities have been conceptualized to have the same outer diameter. The rf frequency, beam entrance and exit tubes and inner conductor size are then selected to form a cavity geometry that will produce the design specifications. A summary of cavity specifications for the three ISAC-II superconducting cavity types are given in Table 1.

Table 1: Geometrical parameters of ISAC-II superconducting cavities used in the RELAX-3D modeling. Lengths in the beam direction are given for entrance and exit grounded tubes, the acceleration gaps and the inner conductor tube.

\begin{tabular}{|l|c|c|c|}
\hline Cavity & Low & Medium & High \\
\hline$\beta(\%)$ & 4.2 & 7.2 & 10.6 \\
f (MHz) & 70.72 & 106.08 & 141.44 \\
ID (mm) & 180 & 180 & 180 \\
Inner Tube L (mm) & 40 & 60 & 60 \\
Gap L (mm) & 46 & 40 & 50 \\
Grounded Tube L (mm) & 24 & 20 & 10 \\
Full Bore (mm) & 20 & 20 & 20 \\
\hline \hline
\end{tabular}


These geometric values are input into RELAX-3D to produce a static electric field distribution. The fields are then time modulated and integrated to calculate the impact on an ion of a given velocity and phase. The cavities will typically be operated at a negative synchronous phase for stable longitudinal motion and this gives rise to a net transverse defocussing force and vertical steering due to the vertical asymmetry. A summary plot of the cavity transverse perturbations for the three geometries are shown in Fig. 3 assuming a phase of $-30^{\circ}$, a field gradient of $6 \mathrm{MV} / \mathrm{m}$ and $A / q$ of 3 .

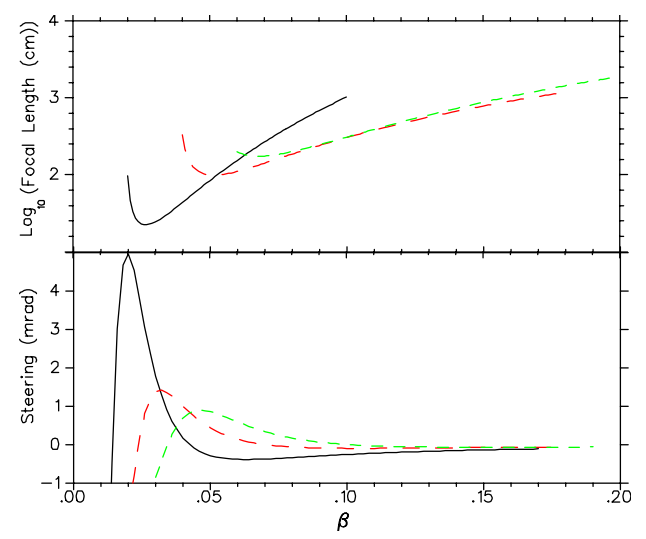

Figure 3: (a) Integrated defocussing effect and (b) steering effect from single cavities and three geometries assuming $6 \mathrm{MV} / \mathrm{m}, A / q=3$, and $\phi_{s}=-30^{\circ}$.

These calculations point out the strong defocussing and steering produced in the low $\beta$ section. The latter is especially troublesome for multi-charge acceleration since the transverse phase advance per cell varies with charge so that vertical deflections on the ensemble will eventually become incoherent and lead to emittance growth. We may, as a result, alter the cavity geometry to lower the design velocity of the low $\beta$ cavity.

\section{MULTI-CHARGE ACCELERATION}

Fig. 4 summarizes a set of possible operating modes that illustrate the benefits of multi-charge acceleration. In the model we include two stripping stages, S1 and S2 (Fig. 1), one at $0.4 \mathrm{MeV} / \mathrm{u}$ and one after the first twelve cavities or after $10 \mathrm{MV}$ of effective accelerating voltage. Fig. 4(a) gives the expected equilibrium charge state for stripping from each of the strippers. Stripping from S2 produces a higher charge state due to the higher incident beam velocity. Beam dynamics simulations, reported below, indicate that we can successfully accelerate particles with a relative charge difference of $\sim 5 \%$ in the low energy section and of $\sim 7 \%$ in the high energy section. These bounds give the number of possible charge states that can be simultaneously accelerated by $\mathrm{S} 1$ and $\mathrm{S} 2$. These are plotted in Fig. 4(b). With multi-charge acceleration an experimenter could add S2 and gain a higher final energy with only minimal intensity loss. The expected final energy of the beam with two stripping foils compared to a single stripper, S1, is shown in Fig. 4(c). A plot of the expected acceleration efficient for the single charge reference design case and for multi-charge acceleration with one or two stripping stages is given in Fig. 4(d). Note that even with two stripping foils the overall efficiency for most ions is improved over the single charge state case.

The drawback of multi-charge acceleration is an increase in the transverse and longitudinal emittance and the increased complexity and cost of the transport and matching sections. If the final energy is sufficient it is conceivable to add a final stripper after acceleration to reach a single fully stripped state. For ISAC-II this would be feasible for ions with $Z \leq 30[5]$.
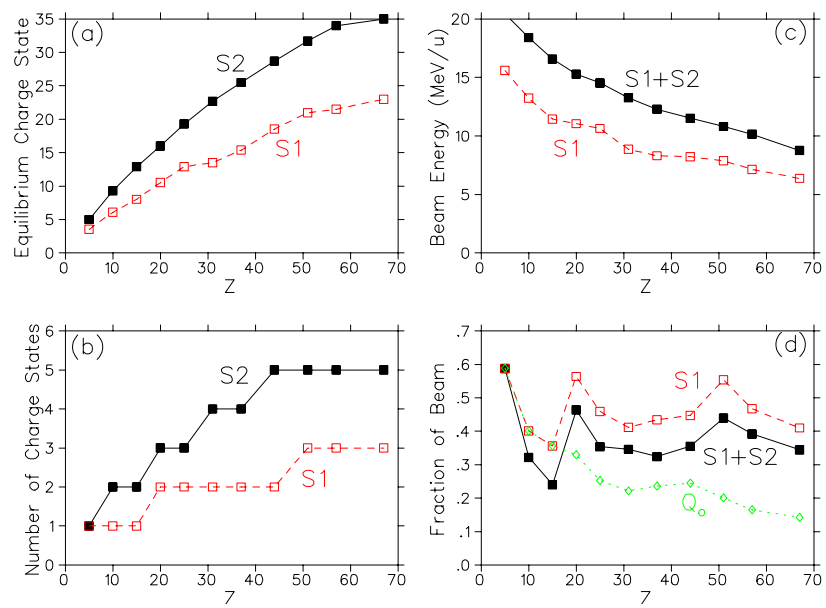

Figure 4: Summary plots for multi-charge acceleration. Plotted in (a) are the equilibrium charge states from a carbon foil at $0.4 \mathrm{MeV} / \mathrm{u}$ and from a second foil, S2, after $10 \mathrm{MV}$ of acceleration. The number of potential charge states that could be simultaneously accelerated are shown in (b). The final energy from S1 alone or S1 and S2 together are given in (c) while the acceleration efficiency for single charge acceleration $\left(Q_{o}\right)$ and multi charge acceleration with $\mathrm{S} 1$ and $\mathrm{S} 2$ are given in (d).

\subsection{Lattice Constraints}

We consider a single non-accelerating cell made with focusing elements, triplets, doublets or solenoids, separated by four accelerating cavities represented as defocussing lenses. The cells are analyzed to find matched solutions for a given phase advance for the reference charge, $q_{0}$ as well as other $q^{\prime}$ values. The difference between the matched solutions for $q_{0}$ and $q^{\prime}$ are used to obtain a mismatch factor to calculate, based on a given cylindrical aperture and beam emittance, the range of charge states that can be transported for the given phase advance, energy range and lattice type.

A phase advance near $90^{\circ}$ was found to maximize the acceptance range. Fig. 5 gives the range of charge states that can be successfully transported for each lattice type as a function of energy for a phase advance of $90^{\circ}$. The 
range of transported charge is very narrow in the low energy section where the high cavity defocussing results in large values of $\beta_{\max }$. It should be noted that the simpified study does not differentiate between magnet bore and linac bore sizes as would occur in a typical linac.

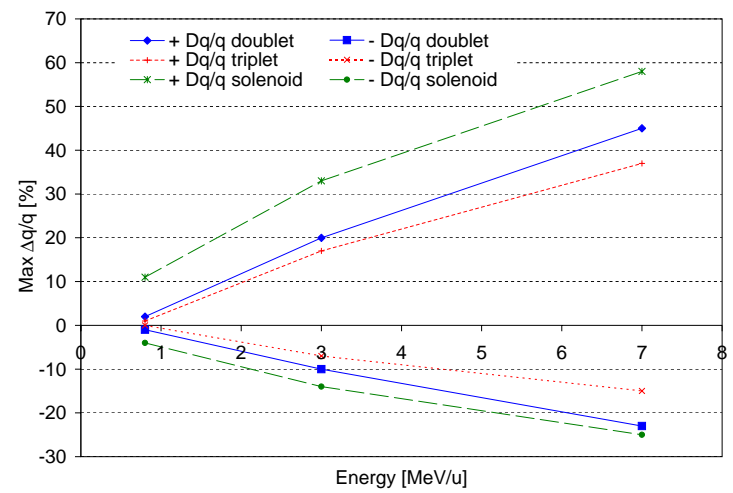

Figure 5: Different charge transported for the three lattices and at different energies

\subsection{Multi-Particle Studies}

The beam simulation code LANA was used to investigate the particular case of ${ }^{132} \mathrm{Sn}$ for a solenoid linac lattice. In the study 5000 particles are accelerated in the simulated ISAC-II linac with stripping at S1 and S2 as defined above. Longitudinal acceptance is strongly influenced by inter-cryostat length. A length of $40 \mathrm{~cm}$ was chosen in the study with a magnetic solenoid positioned every four cavities. Multi-charge simulations were done by fixing the linac parameters for the $q_{o}$ case and altering the charge of the initial beam. Three charge states, 20, 21 and 22, are accelerated after S1 and five charge states, 29-33, are accelerated after $\mathrm{S} 2$. .

Fig. 6 shows the RMS and maximum beam envelopes for both the reference particle and the multi-charge case. The transverse focussing is sufficient to keep the envelope size for all charges to less than double that for a single charge state.

Acceleration of multiple charge states will lead to longitudinal emittance growth of the ensemble since each charge will oscillate around a different synchronous phase with different oscillation amplitudes and phase advance of the constituent charges[3]. Fig. 7 shows the final longitudinal phase space with all the final five charges compared to the longitudinal emittance for the single charge state $q_{0}$. The longitudinal emittance of the ensemble is about a factor of 10 larger than the single charge case.

Magnetic and rf mis-alignments and dipole field components in the cavities produce coherent transverse perturbations. Each charge state will proceed through the linac with a different transverse phase advance leading to emittance growth of the ensemble. Mis-alignment sensitivity is simulated by shifting the initial beam position by $0.5 \mathrm{~mm}$ in both $x$ and $y$. A transverse emittance growth of about a

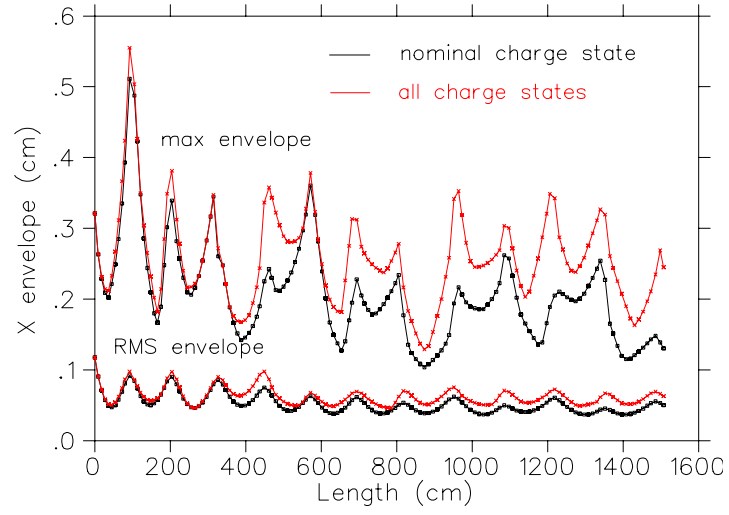

Figure 6: Shown are the RMS and Maximum envelopes for the reference charge state (black) and the combined multicharge state cases (red).

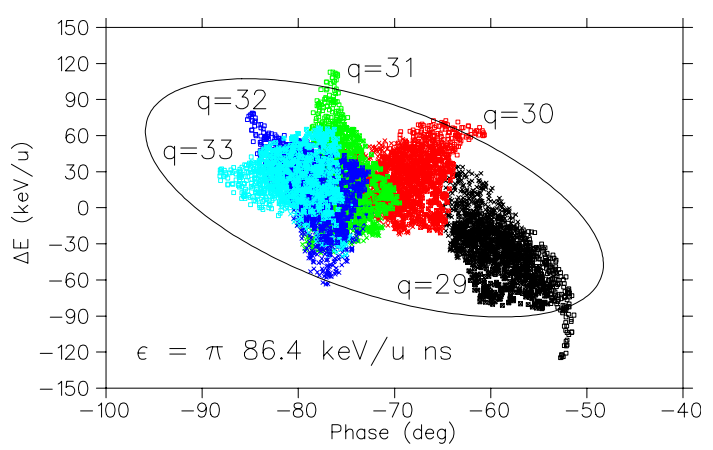

Figure 7: Final longitudinal phase space of (a) the single charge state case and (b) the five different charges after acceleration through the linac with optimized solenoid focussing. The enfolding ellipse in each case is highlighted.

factor of $2-3$ is produced.

Further work will involve adding the realistic fields of the cavities including dipole components into the simulation and adding matching and transport sections to the model.

\section{REFERENCES}

[1] R.E. Laxdal, "Completion and Operations of ISAC-I and Extension to ISAC-II", these proceedings.

[2] A. Facco, et al, "The Superconducting Medium $\beta$ Prototype for radioactive beam acceleration at TRIUMF", these proceedings.

[3] P.N. Ostroumov, et al, "Multiple Charge Beam Dynamics in an Ion Linac", Proc. of the XX International Linac Conference (LINAC2000), Monterey, Aug. 2000, p. 1018.

[4] M. Pasini and R.E. Laxdal, "ISAC II: Beam Studies with Multi-charge Beams", TRIUMF Design Note TRI-DN-0108, April 2001.

[5] K. Shima, et al, "Equilibrium Charge Fractions of Ions of $Z=4-92$ Emerging from a Carbon Foil", Atomic Data and Nuclear Data Tables 51, 173-241 (1992). 